\title{
Algebraic Fractional-Step Schemes for Time-Dependent Incompressible Navier-Stokes Equations
}

\author{
Paola Gervasio $^{1}$ and Fausto Saleri $^{2}$
}

Received October 22, 2004; accepted (in revised form) October 7, 2005; Published online March 13, 2006

\begin{abstract}
The numerical investigation of a recent family of algebraic fractional-step methods (the so called Yosida methods) for the solution of the incompressible time-dependent Navier-Stokes equations is presented. A comparison with the Karniadakis-Israeli-Orszag method Karniadakis et al. (1991, J. Comput. Phys. 97, 414-443) is carried out. The high accuracy in time of these schemes well combines with the high accuracy in space of spectral methods.
\end{abstract}

KEY WORDS: Navier-Stokes equations; algebraic fractional step methods; spectral methods.

\section{INTRODUCTION}

The algebraic fractional step methods are based on the decomposition of the matrix associated with the global discretization (in both space and time) of the Navier-Stokes equations. This decomposition (or splitting) could be performed by either a sum of simpler matrices, or a product of block-triangular matrices, as Perot proposed in [8]. Perot revisited the Chorin-Temam method by noting that, by loosing the boundary conditions, the discretized projection step operator was the product between a lower block-triangular matrix $L$ and an upper block-triangular matrix $U$, so that to do a step of the Chorin-Temam method was equivalent to compute the matrices $L$ and $U$ and to solve two block-triangular systems. With this approach the boundary conditions were incorporated in

\footnotetext{
${ }^{1}$ Department of Mathematics, University of Brescia, 25133 Brescia, Italy.

E-mail: gervasio@ing.unibs.it

${ }^{2}$ Department of Mathematics, MOX, Politecnico di Milano, 20133 Milano, Italy.

E-mail: fausto.saleri@polimi.it
} 
the discretized operator and all was performed from an algebraic point of view. This re-interpretation of the Chorin-Temam scheme gave rise to the investigation of new families of (algebraic) fractional step schemes, which can have not a differential counterpart but have good accuracy and stability properties. To set up new methods, the idea is to act on the choice of the factors $L$ and $U$ of the inexact factorization. The Yosida method, proposed in $[9,10]$, is based on such an idea. Two more accurate versions have been presented in [11] and [3, 4]. These three different forms of the Yosida scheme (named Yosida, Yosida3 and Yosida4) introduce a splitting error on the velocity of order $2-4$, respectively, with respect to the time step $\Delta t$, so that we can take advantage of high accuracy in both time and space if we couple them with spectral methods. Nevertheless, Yosida methods can be coupled with other approximation methods like finite elements or finite differences and, as a matter of facts, they have been proposed in $[9,10]$ in conjunction with finite elements.

In this paper we compare two schemes of Yosida type with two schemes belonging to the family of stiffly stable Karniadakis-IsraeliOrszag (KIO) methods.

\section{DEFINITIONS AND SETTINGS}

We consider the Navier-Stokes equations for Newtonian incompressible fluids in the primal velocity-pressure formulation. Let $\Omega \subset \mathbb{R}^{2}$ be an open bounded domain with a Lipschitz boundary, and $T \in \mathbb{R}^{+}$. Given an external field $\mathbf{f} \in\left[L^{2}\left(0, T, L^{2}(\Omega)\right)\right]^{2}$, a boundary data $\mathbf{g} \in$ $\left[L^{2}\left(0, T, H^{1 / 2}(\partial \Omega)\right)\right]^{2}$ and an initial datum $\mathbf{u}_{0} \in\left[H^{1}(\Omega)\right]^{2}$ such that $\nabla \cdot \mathbf{u}_{0}=0$, we look for the velocity field $\mathbf{u} \in\left[L^{2}\left(0, T, H^{1}(\Omega)\right)\right]^{2}$ and the pressure field $p \in L^{2}\left(0, T, L_{0}^{2}(\Omega)\right)$ solutions of

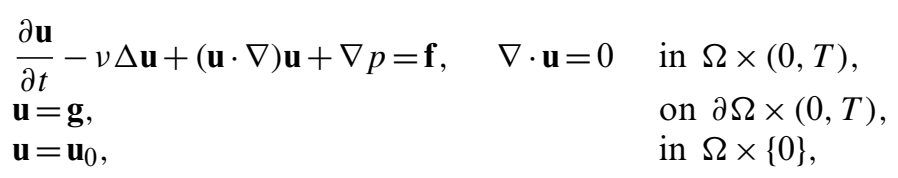

where $v=$ const. $>0$ is the kinematic viscosity. It is well known that problem (1) admits a unique solution.

We approximate in time the Navier-Stokes system (1) by a Backward Differentiation Formula (BDF) of order $q(q=1,2,3)$ and we linearize the convective term by an extrapolation formula of the same order $q$.

Given $\Delta t \in(0, T)$, we set $t^{0}=0$ and $t^{n}=t^{0}+n \Delta t$ with $n=1, \ldots, N_{T}$ and $N_{T}=\left[\frac{T}{\Delta t}\right]$. Therefore, for any $n \geqslant n_{0}=q-1$, we look for the solution 
$\left(\mathbf{u}^{n+1}, p^{n+1}\right)$ of the system

$$
\begin{aligned}
\frac{\beta_{-1}}{\Delta t} \mathbf{u}^{n+1}-v \Delta \mathbf{u}^{n+1}+\left(\mathbf{u}^{*} \cdot \nabla\right) \mathbf{u}^{n+1}+\nabla p^{n+1}=\tilde{\mathbf{f}}^{n+1}, & & \text { in } \Omega, \\
\nabla \cdot \mathbf{u}^{n+1}=0, & & \text { in } \Omega, \\
\mathbf{u}^{n+1}=\mathbf{g}^{n+1}, & & \text { on } \partial \Omega,
\end{aligned}
$$

where $\beta_{-1}$ is the leading coefficient of BDF of order $q, \mathbf{u}^{*}=\sum_{j=0}^{q-1} \alpha_{j} \mathbf{u}^{n-j}$ $\left(\alpha_{j}\right.$ are the coefficients of the extrapolation formula of order $q$ ) and $\tilde{\mathbf{f}}^{n+1}=$ $\mathbf{f}^{n+1}+\frac{1}{\Delta t} \sum_{j=0}^{q-1} \beta_{j} \mathbf{u}^{n-j}\left(\beta_{j}\right.$ are the coefficients of BDF of order $\left.q\right)$.

For BDF schemes with order greater than 1 we need further initial data that in our cases will be provided by the exact solution. For general cases, initial data could be provided by suitable explicit schemes (e.g. Runge-Kutta) of the same order of the BDF used.

For the discretization in space, we chose conforming spectral elements with numerical integration of Gauss-Legendre type.

In order to overcome instabilities due to the mixed formulation of Navier-Stokes equations we make use of the $\mathbb{Q}_{N}-\mathbb{Q}_{N-2}$ scheme with staggered grids [1], according to which local polynomials of degree $N$ in each variable are used to approximate the velocity field and local polynomials of degree $N-2$ in each variable are used to approximate the pressure. By this choice, the inf-sup condition is satisfied through a constant which is proportional to $N^{-1 / 2}[1]$.

At each time-step $t_{n} \in(0, T]$ we look for the numerical solution $\left(\mathbf{u}_{\mathcal{H}}^{n+1}, p_{\mathcal{H}}^{n+1}\right) \in \mathbf{V}_{\mathcal{H}} \times Q_{\mathcal{H}}$, where $\mathbf{V}_{\mathcal{H}}:=\left[\mathbb{Q}_{\mathcal{H}}(\Omega) \cap H_{0}^{1}(\Omega)\right]^{2}$ and $Q_{\mathcal{H}}:=\left\{q_{\mathcal{H}} \in\right.$ $\left.L_{0}^{2}(\Omega): q_{\mathcal{H} \mid T_{k}} \in \mathbb{Q}_{N-2}\left(T_{k}\right), \forall T_{k} \in \mathcal{T}_{h}\right\}$ are the finite dimensional spaces, $\mathcal{T}_{h}$ is a suitable partition of $\Omega$ in $N_{e}$ quadrilaterals $T_{k}$ and $\mathbb{Q}_{\mathcal{H}}:=\left\{v_{\mathcal{H}} \in C^{0}(\bar{\Omega})\right.$ : $\left.v_{\mathcal{H} \mid T_{k}} \in \mathbb{Q}_{N}\left(T_{k}\right), \forall T_{k} \in \mathcal{T}_{h}\right\}$.

We denote by $\mathbf{U}^{n} \in \mathbb{R}^{2 N_{v}}$ the array of the velocity grid function $\mathbf{u}_{\mathcal{H}}^{n+1}$ evaluated on the Gauss-Legendre-Lobatto nodes at time $t^{n}$, and by $\mathbf{P}^{n} \in$ $\mathbb{R}^{N_{p}}$ the array of the pressure grid function $p_{\mathcal{H}}^{n+1}$ evaluated on the GaussLegendre nodes at time $t^{n}$. We reduce the system to the unknowns which are not associated to Dirichlet boundary conditions and the right hand side is modified accordingly, taking into account the contributions that the Dirichlet boundary nodes give to internal nodes. This step generates a right hand side $\left[\mathbf{F}_{1}^{n+1}, \mathbf{F}_{2}^{n+1}\right]^{T}$ that is non zero also in the continuity equation.

We denote by $M$ the mass matrix, by $K$ the stiffness matrix, by $B$ (resp. by $N\left(\mathbf{U}^{*}\right)$ ) the matrix related to the discretization of $-\nabla \cdot$ (resp. $\left(\mathbf{U}^{*}\right.$. $\nabla) \cdot)$, and we set $C=\frac{\beta_{-1}}{\Delta t} M+v K+N\left(\mathbf{U}^{*}\right)$, then the full approximation of 
system (2) reads

$$
\begin{gathered}
C \mathbf{U}^{n+1}+B^{T} \mathbf{P}^{n+1}=\mathbf{F}_{1}^{n+1} \quad \text { or } \quad A \mathbf{W}^{n+1}=\mathbf{F}^{n+1} \\
B \mathbf{U}^{n+1}=\mathbf{F}_{2}^{n+1} \\
\text { with } A=\left[\begin{array}{cc}
C & B^{T} \\
B & 0
\end{array}\right], \quad \mathbf{W}^{n+1}=\left[\begin{array}{l}
\mathbf{U}^{n+1} \\
\mathbf{P}^{n+1}
\end{array}\right], \quad \mathbf{F}^{n+1}=\left[\begin{array}{l}
\mathbf{F}_{1}^{n+1} \\
\mathbf{F}_{2}^{n+1}
\end{array}\right] .
\end{gathered}
$$

To solve system (3) one could use a global approach such as a preconditioned Krylov method with either algebraic or differential preconditioners or again with Schwarz type domain decomposition preconditioners or a pressure Schur complement approach.

Alternatively, system (3) can be solved by a block $L U$ factorization with

$$
L=\left[\begin{array}{cc}
C & 0 \\
B & -B C^{-1} B^{T}
\end{array}\right], \quad U=\left[\begin{array}{cc}
I & C^{-1} B^{T} \\
0 & I
\end{array}\right]
$$

The matrix $\Sigma:=-B C^{-1} B^{T}$ is the so called pressure Schur complement matrix and to solve system (3) through the block $L U$ factorization (4) consists in finding the solution of the following subsystems (from now on we will drop the index $n+1$ from all the vectors $\left.\mathbf{U}^{n+1}, \mathbf{P}^{n+1}, \mathbf{W}^{n+1}, \mathbf{F}^{n+1}\right)$ :

$$
\begin{aligned}
& L-\text { step: find } \widetilde{\mathbf{U}}, \widetilde{\mathbf{P}}:\left\{\begin{array}{l}
C \widetilde{\mathbf{U}}=\mathbf{F}_{1} \\
\Sigma \widetilde{\mathbf{P}}=\mathbf{F}_{2}-B \widetilde{\mathbf{U}}
\end{array}\right. \\
& U-\text { step : find } \mathbf{U}, \mathbf{P}:\left\{\begin{array}{l}
\mathbf{P}=\widetilde{\mathbf{P}} \\
C(\widetilde{\mathbf{U}}-\mathbf{U})=B^{T} \mathbf{P}
\end{array}\right.
\end{aligned}
$$

It is essential to note that, since a semi-implicit treatment of the convective term is considered, matrix $C$ is time-dependent and the resolution of the system involving $\Sigma$, either by a direct method or an iterative one, is quite expensive. The alternative is to replace $\Sigma$ by a suitable approximation: this is the idea which the Yosida scheme is based on.

\section{THE YOSIDA SCHEME AND ITS IMPROVED VERSIONS}

The Yosida scheme is an algebraic fractional-step scheme, it was introduced in $[9,10]$ and it is characterized by the fact that the exact $L U$ factorization of the matrix $A$ is replaced by an inexact $L U$ factorization. In other words, the Schur complement $\Sigma$ is replaced by a suitable matrix $S$. Such a scheme differs from the algebraic version of Chorin-Temam scheme (see $[2,8]$ ) basically for the fact that it is momentum preserving, while the Chorin-Temam scheme is mass preserving. The Yosida scheme introduces 
a splitting error for the velocity that is $\mathcal{O}\left(\Delta t^{2}\right)$ and, if it is coupled with BDF2 (BDF-Backward Differentiation Formulas - of order 2), the resulting approximation scheme is $\mathcal{O}\left(\Delta t^{2}\right)$, too. The splitting error on the pressure is $\mathcal{O}\left(\Delta t^{3 / 2}\right)[3,4]$.

In $[4,11]$ two more accurate versions of Yosida method are proposed and analyzed. Such improved Yosida schemes introduce a new matrix $Q$, inside the $U$-step and acting on the pressure, which "fixes" the approximation of the Schur complement $\Sigma$. All is done at the algebraic level, without a compulsory differential interpretation and this feature allows us to neglect the setting up of boundary conditions for the sub-steps. At each time step, we look for $\widehat{\mathbf{W}}^{n+1}=\left[\widehat{\mathbf{U}}^{n+1}, \widehat{\mathbf{P}}^{n+1}\right]^{T}$, approximation of $\mathbf{W}^{n+1}$ and solution of $\hat{A} \widehat{\mathbf{W}}^{n+1}=\widehat{\mathbf{F}}^{n+1}$, where $\hat{A}=\hat{L} \hat{U}$ is the inexact factorization of $A$ and $\widehat{\mathbf{F}}^{n+1}$ is the perturbed right hand side which takes into account the approximate solutions of previous steps.

The factors $\hat{L}$ and $\hat{U}$ are defined as follows:

$$
\hat{L}=\left[\begin{array}{cc}
C & 0 \\
B & -B H B^{T}
\end{array}\right], \quad \hat{U}=\left[\begin{array}{cc}
I & C^{-1} B^{T} \\
0 & Q
\end{array}\right] \quad \text { with } H:=\frac{\Delta t}{\beta_{-1}} M^{-1}
$$

and, if $Q=I$ we recover the Yosida scheme, if $Q$ is such that $Q^{-1}=$ $-S^{-1} D$ with $D=B H C H B^{T}$ we have the so called Yosida3 scheme, while if $Q$ is such that $Q^{-1}=-S^{-1} D+\left(S^{-1} D\right)^{2}+S^{-1} B(H C)^{2} H B^{T}$, we have the so called Yosida4 scheme.

To solve the system $\hat{A} \widehat{\mathbf{W}}=\widehat{\mathbf{F}}$ at a generic time step, means to solve the following subsystems:

$$
\begin{aligned}
& L-\text { step: find } \widetilde{\mathbf{U}}, \widetilde{\mathbf{P}}:\left\{\begin{array}{l}
C \widetilde{\mathbf{U}}=\widehat{\mathbf{F}}_{1} \\
S \widetilde{\mathbf{P}}=\widehat{\mathbf{F}}_{2}-B \widetilde{\mathbf{U}}
\end{array}\right. \\
& U-\text { step: find } \widehat{\mathbf{U}}, \widehat{\mathbf{P}}:\left\{\begin{array}{l}
Q \widehat{\mathbf{P}}=\widetilde{\mathbf{P}} \\
C(\widetilde{\mathbf{U}}-\widehat{\mathbf{U}})=B^{T} \widehat{\mathbf{P}}
\end{array}\right.
\end{aligned}
$$

where the matrix $S:=-B H B^{T}$ is an approximation of the Schur complement $\Sigma$ and it can be interpreted as the Neumann expansion of order zero of the inverse of $\Sigma$.

Under suitable stability conditions on the time-step [3], the scheme Yosida3 is third-order accurate in time for the velocity and of order 5/2 for the pressure, provided it is associated with a third-order BDF scheme, while the scheme Yosida4 is fourth-order accurate in time for the velocity and of order $7 / 2$ for the pressure, provided it is associated with a fourth-order BDF scheme. The detailed description of the last method, such as its analysis, is presented in [3, 4].

We note that the schemes Yosida3 and Yosida4 differ from the algebraic fractional-step proposed by Couzy [2] for two reasons. The first 
one, as said at the beginning of this section, is that Yosida schemes are momentum preserving while the schemes proposed by Couzy are based on the Chorin-Temam method, and therefore they are mass preserving. The second reason is that Couzy improves the order of the scheme by approximating the matrix $C^{-1}$ with a Taylor expansion of order 3 (he notes that the expansion of even order are unstable), while in the Yosida schemes we don't change the approximation of $C^{-1}$ (it is always $H$ ), but we introduce a new matrix $Q$, acting on the pressure, in order to have $\|\Sigma-S Q\|=$ $\mathcal{O}\left(\Delta t^{q}\right)$, with $q=3,4$.

Remark 3.1. Even though the splitting error of Yosida scheme on the velocity is $\mathcal{O}\left(\Delta t^{2}\right)$, it is higher than the error induced by BDF2, so that, if we want that the splitting error does not prevail over the BDF-error for $\Delta t \rightarrow 0$, it is better to join Yosida with BDF1. The same arguments apply to both Yosida3 and Yosida4: we will join Yosida3 with BDF2 and Yosida4 with BDF3.

The computational effort required by either Yosida3 or Yosida4 methods increases mildly with respect to the cost of Yosida. As a matter of fact, at each time-step we have to solve an additional linear system of type $Q \widehat{\mathbf{P}}=\tilde{\mathbf{P}}$ in comparison with Yosida. In [3] a comparison between the computational cost of Yosida, Yosida3, and Yosida4 is carried out, as well as a comparison with the computational cost of the unsplit approach.

\section{KIO METHOD}

In this paper we want to compare Yosida schemes with the stiffly stable version of the KIO method. For $\mathbf{f} \equiv \mathbf{0}$ in $\Omega$ and $(\mathbf{g} \cdot \mathbf{n})_{\mid \partial \Omega}=0$ the scheme reads ([6, Sec. 4.2]):

$$
\begin{aligned}
& \text { 1. } \frac{\hat{\mathbf{v}}}{\Delta t}=\sum_{j=0}^{q-1} \beta_{j} \mathbf{u}^{n-j}-\sum_{j=0}^{q-1} \alpha_{j} N\left(\mathbf{u}^{n-j}\right) \mathbf{u}^{n-j} \quad \text { in } \Omega \cup \partial \Omega \\
& \text { 2. } \begin{cases}\Delta p^{n+1}=\nabla \cdot\left(\frac{\hat{\mathbf{v}}}{\Delta t}\right) & \text { in } \Omega \\
\frac{\partial p^{n+1}}{\partial n}=\mathbf{n} \cdot\left(-v \sum_{j=0}^{q-1} \alpha_{j} \nabla \times\left(\nabla \times \mathbf{u}^{n-j}\right)\right) & \text { on } \partial \Omega\end{cases} \\
& \text { 3. } \frac{\hat{\mathbf{v}}}{\Delta t}=\frac{\hat{\mathbf{v}}}{\Delta t}-\nabla p^{n+1} \quad \text { in } \Omega \cup \partial \Omega \\
& \text { 4. } \begin{cases}\frac{\beta_{-1} \mathbf{u}^{n+1}-\hat{\mathbf{v}}}{\Delta t}=v \Delta \mathbf{u}^{n+1} & \text { in } \Omega \\
\mathbf{u}^{n+1}=\mathbf{0} & \text { on } \partial \Omega,\end{cases}
\end{aligned}
$$

where the coefficients $\alpha_{j}$ and $\beta_{j}$ have the same meaning given in (2). Numerical results in (7) show that, if one uses the BDF scheme and the 
extrapolation formula of the same order $q$, than also KIO method is accurate up to order $q$ in time, thanks also to the use of the rotational treatment of the diffusive term to set up the boundary condition on the pressure.

The scheme (7) can be generalized for any external force field $\mathbf{f} \neq \mathbf{0}$ and any boundary data $\mathbf{g}:(\mathbf{g} \cdot \mathbf{n})_{\mid \partial \Omega} \neq 0$ by replacing the first two steps of (7) with the following ones:

1a. $\frac{\hat{\mathbf{v}}}{\Delta t}=\sum_{j=0}^{q-1} \beta_{j} \mathbf{u}^{n-j}-\sum_{j=0}^{q-1} \alpha_{j} N\left(\mathbf{u}^{n-j}\right) \mathbf{u}^{n-j}+\mathbf{f}^{n+1}$
2a. $\begin{cases}\Delta p^{n+1}=\nabla \cdot\left(\frac{\hat{\mathbf{v}}}{\Delta t}\right) & \text { in } \Omega \cup \partial \Omega \\ \frac{\partial p^{n+1}}{\partial n}=\mathbf{n} \cdot\left(\frac{\hat{\mathbf{v}}}{\Delta t}-v \sum_{j=0}^{q-1} \alpha_{j} \nabla \times\left(\nabla \times \mathbf{u}^{n-j}\right)\right) & \text { on } \partial \Omega\end{cases}$

and by imposing non-homogeneous boundary conditions on $\mathbf{u}^{n+1}$ inside step 4.

Remark 4.1. We note that if we want to approximate the NavierStokes equation by conformal spectral element methods of $\mathbb{Q}_{N}-\mathbb{Q}_{N-2}$ type, with discontinuous pressure, the third step of KIO method has to be stabilized following the ideas of Houston et al. [5]. On the contrary, being the Yosida scheme of algebraic type, no trouble, in dealing with discontinuous pressure, is present.

\section{NUMERICAL RESULTS}

We define the errors between the exact solution of Navier-Stokes equations and the numerical one (obtained by either Yosida or KIO method) as

$$
E_{\mathbf{u}}=\left(\Delta t \sum_{n=0}^{N_{T}}\left\|\mathbf{u}\left(t_{n}\right)-\mathbf{u}_{\mathcal{H}}^{n}\right\|_{H^{1}(\Omega)}^{2}\right)^{1 / 2}, \quad E_{p}=\left(\Delta t \sum_{n=0}^{N_{T}}\left\|p\left(t_{n}\right)-p_{\mathcal{H}^{\prime}}^{n}\right\|_{L^{2}(\Omega)}^{2}\right)^{1 / 2}
$$

Test case \#1. First of all we consider a test case with computational domain $\Omega=(-0.5,0.5)^{2}$ and $t \in(0, T)=(0,1), \mathbf{f} \equiv 0$ in $\Omega \times(0, T)$ and $\mathbf{u} \cdot \mathbf{n}=$ 0 on $\partial \Omega \times(0, T)$. A solution that satisfies these properties is the so-called Kim and Moin solution [7]:

$$
\begin{aligned}
& \mathbf{u}(x, y, t)=e^{\left(-2 \alpha^{2} \pi^{2} t \nu\right)}[-\cos (\alpha \pi x) \sin (\alpha \pi y), \sin (\alpha \pi x) \cos (\alpha \pi y)]^{T}, \\
& p(x, y, t)=-\frac{1}{4}[\cos (2 \alpha \pi x)+\cos (2 \alpha \pi y)] e^{\left(-4 \alpha^{2} \pi^{2} t \nu\right)}
\end{aligned}
$$



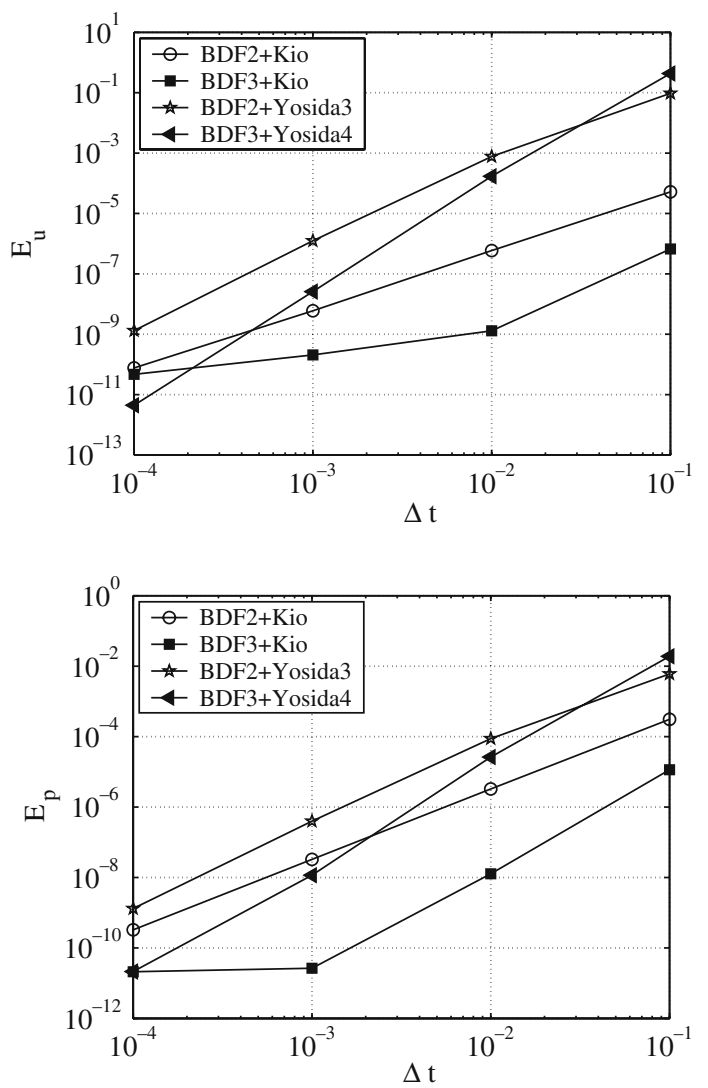

Fig. 1. The errors $E_{\mathbf{u}}, E_{p}$ for the test case $\# 1$.

with $\alpha \in \mathbb{R}^{+}$. We have chosen $\alpha=1$ and $\nu=10^{-2}$. By taking the period of the solution as reference length, it follows that the Reynolds number is $R e=200$. In Fig. 1 we show the errors $E_{\mathbf{u}}, E_{p}$ versus the time step $\Delta t$ for a space discretization with $N_{e}=1$ and $N=16$, while in Fig. 2 we show the behavior of $\left\|\mathbf{u}\left(t_{n}\right)-\mathbf{u}_{\mathcal{H}}^{n}\right\|_{H^{1}(\Omega)}$, of $\left\|p\left(t_{n}\right)-p_{\mathcal{H}}^{n}\right\|_{L^{2}(\Omega)}$ and of $\left\|\nabla \cdot \mathbf{u}_{\mathcal{H}}^{n}\right\|_{L^{\infty}(\Omega)}$ versus time. We note that KIO methods perform better than Yosida when we use BDF2, or when we use BDF3 with not too small time-step.

Test case \#2. We consider now the computational domain $\Omega=(-1,1)^{2}$ and $t \in(0, T)=(0,1)$, with the right hand side $\mathbf{f}$ and the boundary data such that the exact solution of Navier-Stokes equations is

$$
\begin{aligned}
& \mathbf{u}(x, y, t)=[\sin (x) \sin (y+t), \cos (x) \cos (y+t)]^{T}, \\
& p(x, y, t)=\cos (x) \sin (y+t) .
\end{aligned}
$$



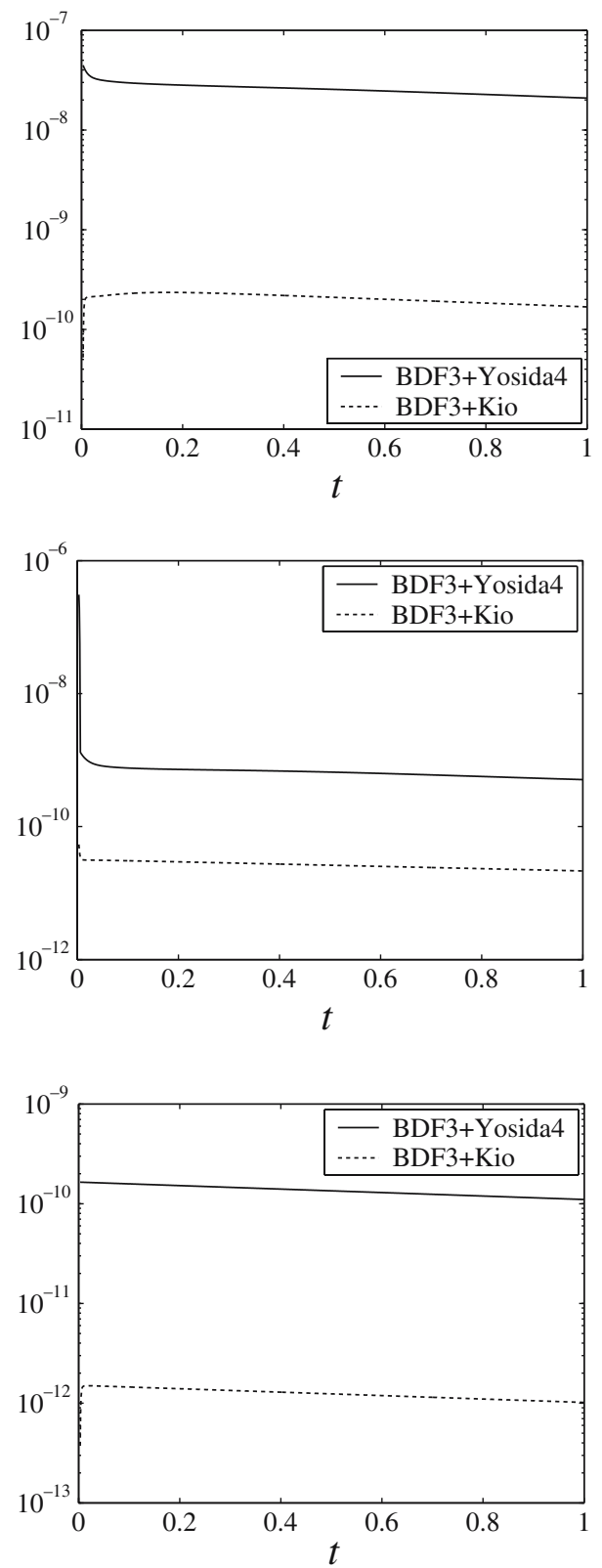

Fig. 2. Evolution in time of the $H^{1}(\Omega)$-norm error for the velocity (top), $L^{2}(\Omega)$-norm error for the pressure (middle) and of the $L^{\infty}(\Omega)$-norm of the divergence of the velocity (bottom) for test case \#1 and BDF3 with $\Delta t=10^{-3}$. 

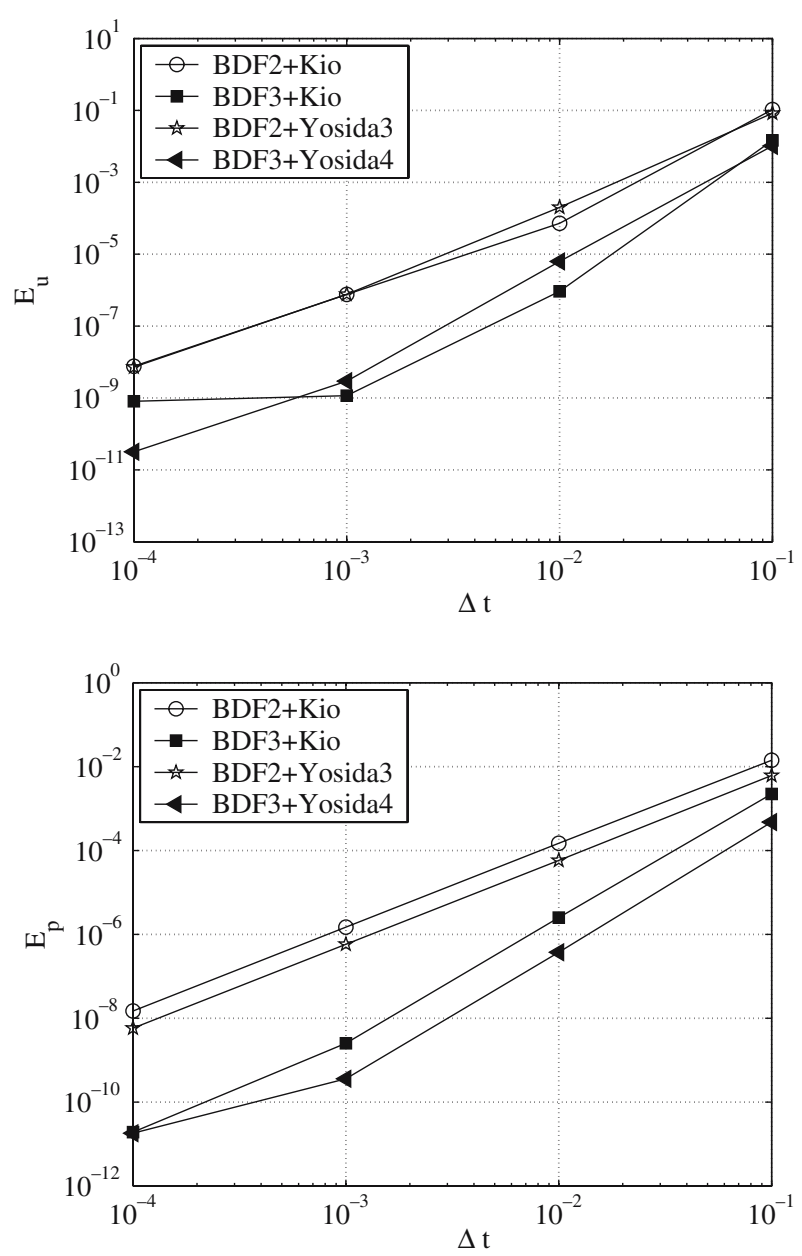

Fig. 3. The errors $E_{\mathbf{u}}, E_{p}$ for the test case \#2.

We note that here $\mathbf{f} \not \equiv \mathbf{0}$ and $(\mathbf{u} \cdot \mathbf{n})_{\mid \partial \Omega} \not \equiv 0$. In Fig. 3 we show the errors $E_{\mathbf{u}}, E_{p}$ versus the time step $\Delta t$ for a space discretization with $N_{e}=1$ and $N=16$ and viscosity $v=10^{-5}$. By taking the period of the solution as reference length, it follows that the Reynolds number is $R e \simeq 60,000$. If we compare the errors $E_{\mathbf{u}}, E_{p}$ we can conclude that Yosida and KIO methods behave in a similar manner. Nevertheless, if we consider long-time computations, we observe that Yosida schemes seem more stable than KIO. In Figure 4 we show the behavior of $\left\|\mathbf{u}\left(t_{n}\right)-\mathbf{u}_{\mathcal{H}}^{n}\right\|_{H^{1}(\Omega)}$, of $\left\|p\left(t_{n}\right)-p_{\mathcal{H}}^{n}\right\|_{L^{2}(\Omega)}$ 

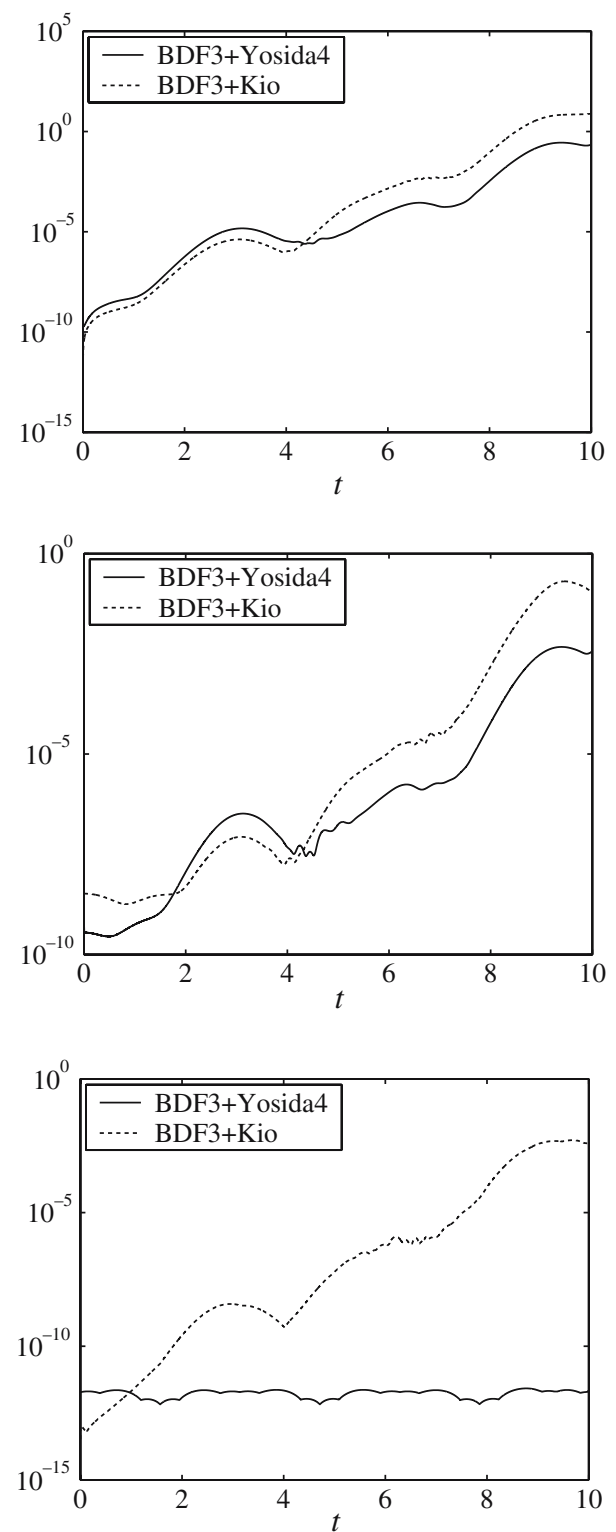

Fig. 4. Evolution in time of the $H^{1}(\Omega)$-norm error for the velocity (top), $L^{2}(\Omega)$-norm error for the pressure (middle) and of the $L^{\infty}(\Omega)$-norm of the divergence of the velocity (bottom) for test case \#2 and BDF3 with $\Delta t=10^{-3}$. 

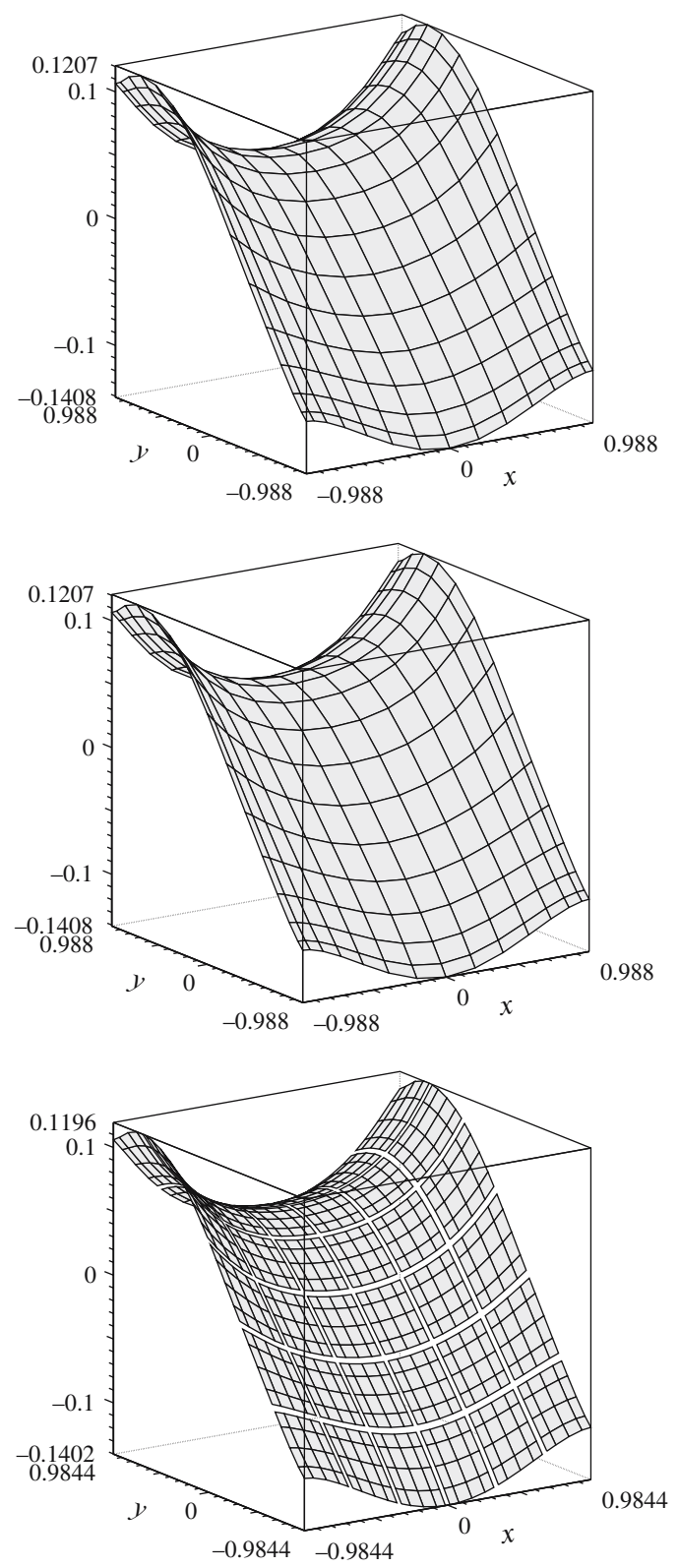

Fig. 5. The error field for the pressure (9) at $T=1$ for BDF3 + Kio with $N_{e}=1, N=16$ (top), BDF3+ Yosida4 with $N_{e}=1, N=16$ (middle), BDF3+ Yosida4 with $N_{e}=6 \times 6, N=6$ (bottom). The viscosity is $v=10^{-2}$, the time-step is $\Delta t=10^{-3}$. 
and of $\left\|\nabla \cdot \mathbf{u}_{\mathcal{H}}^{n}\right\|_{L^{\infty}(\Omega)}$ versus time $t \in(0,10)$ and we note that the norm $\left\|\nabla \cdot \mathbf{u}_{\mathcal{H}}^{n}\right\|_{L^{\infty}(\Omega)}$ for the KIO solution shows sensible growth. It is well known that $\mathrm{KIO}$ is a mass preserving method, nevertheless the magnitude of the boundary divergence flux is controlled directly by the time-step (see [12]) and, for long-time computation, an accumulation of errors could occur as Fig. 4 shows. The numerical results refer to BDF3, but the same conclusions are obtained with BDF2, too.

Finally, in Fig. 5 we show the error field for the pressure on the test solution (9) with viscosity $v=10^{-2}$, for both BDF3+Yosida4 and BDF3+KIO. We see that no boundary layers on the pressure are present for both schemes. Note that we have produced the results for a "large" value of the viscosity in order to remove the suspicion that when the viscosity is too small possible boundary layers on the pressure could be hidden from the small value of the viscosity itself. In view of Remark 4.1 we have no results for Kio method for spectral elements discretization.

\section{REFERENCES}

1. Bernardi, C., and Maday, Y. (1992). Approximations Spectrales de Problèmes aux Limites Elliptiques, Springer Verlag, Paris.

2. Couzy, W. (1995). Spectral Element Discretization of the Unsteady Navier-Stokes Equations and its Iterative Solution on Parallel Computers, Ph.D. thesis, EPFL, Lausanne.

3. Gervasio, P., Saleri, F., and Veneziani, A. (2004). Algebraic fractional step schemes with spectral methods for the incompressible Navier-Stokes equations. Technical report, Seminario Matematico di Brescia, n. 02/04, Brescia, Italy. Accepted for publication in Journal of Computational Physics.

4. Gervasio, P., Saleri, F., and Veneziani, A. (2006). Analysis of high order algebraic fractional step schemes for spectral methods. In preparation.

5. Houston, P., Schwab, C., and Süli, E. (2002). Discontinuous $h p$-finite element methods for advection-diffusion-reaction problems. SIAM J. Numer. Anal. 39(6), 2133-2163.

6. Karniadakis, G. E., Israeli, M., and Orszag, S. A. (1991). High order splitting methods for the incompressible Navier-Stokes equations. J. Comput. Phys. 97, 414-443.

7. Kim, J., and Moin, P. (1985). Application of a fractional-step method to incompressible Navier-Stokes equations. J. Comput. Phys. 59, 308-323.

8. Perot, B. (1993). An analysis of the fractional step method. J. Comp. Phys. 108, 51-58.

9. Quarteroni, A., Saleri, F., and Veneziani, A. (1999). Analysis of the Yosida method for the incompressible Navier-Stokes equations. J. Math. Pures Appl. (9), 78(5), 473-503.

10. Quarteroni, A., Saleri, F., and Veneziani, A. (2000). Factorization methods for the numerical approximation of Navier-Stokes equations. Comput. Methods Appl. Mech. Engrg. 188(1-3), 505-526.

11. Saleri, F., and Veneziani, A. (2005). Pressure-correction algebraic splitting methods for the incompressible navier-stokes equations. SIAM J. Numer. Anal. 43(1), 174-194.

12. Sherwin, S. J., and Karniadakis, G. E. (1999). Spectrallhp Element Methods for CFD, Oxford University Press, Oxford. 